\title{
Normal Sonographic Anatomy of the Wrist With Emphasis on Assessment of Tendons, Nerves, and Ligaments
}

\author{
Salvatore Gitto, MD, Ferdinando Draghi, MD
}

Received July 2, 2015, from the Radiology Institute, Scientific Institute for Research, Hospitalization, and Health Care, Policlinico SanMatteo Foundation, University of Pavia, Pavia, Italy. Revision requested August 6, 2015. Revised manuscript accepted for publication August 24, 2015.

Address correspondence to Salvatore Gitto, MD, Radiology Institute, Scientific Institute for Research, Hospitalization, and Health Care, Policlinico San Matteo Foundation, University of Pavia, Viale Camillo Golgi 19, 27100 Pavia, Italy.

E-mail:sal.gitto@gmail.com
Sonography allows for rapid, cost-effective, noninvasive, and dynamic evaluation of soft tissue structures, thus representing a valuable tool for ruling out musculoskeletal disorders of the wrist. Because of the complexity of the wrist joint, sonographic training and familiarity with normal and variant anatomy are needed to avoid misdiagnosis and improper treatment. The aim of this article is to enlighten readers about the structures representing normal findings or common variants during sonographic evaluations of the wrist. The main text reviews the pertinent gross anatomy and procedures that are recommended to assess the soft tissue structures of the wrist, with particular emphasis given to tendons, nerves, and ligaments. Detailed explanations of the scanning techniques and sonographic appearance of the wrist structures are provided in the figure legends.

Key Words_ligaments; musculoskeletal ultrasound; nerves; sonography; tendons; wrist imaging
1 painful or disabled wrist often compromises daily life activities. It may occur as a result of disorders affecting the tendons, nerves, or ligaments, which require early diagnosis to ensure optimal clinical management. ${ }^{1,2}$ As a cost-effective, quick, and noninvasive imaging technique that also provides dynamic assessment, sonography is a valuable tool for examining the musculoskeletal system. Current ultrasound systems allow for visualization of the small superficial structures of the wrist with high resolution. ${ }^{3,4}$ Since the wrist joint is complex, to avoid misdiagnosis, examiners should be familiar with normal findings and variants commonly depicted when evaluating the wrist. Here we review the normal and variant anatomy of the wrist and discuss the scanning techniques that are recommended to assess the soft tissue structures of the wrist, with particular emphasis given to tendons, nerves, and ligaments. 


\section{Scanning Technique}

High-resolution linear array transducers $(\geq 12 \mathrm{MHz})$ with a broad bandwidth are advisable for a complete evaluation of wrist structures. ${ }^{5}$ All of the images presented in this article were obtained with a high-resolution 12-18-MHz linear transducer (EPIQ7 ultrasound system; Philips Healthcare, Eindhoven, the Netherlands).

The patient needs to sit opposite the operator with the hand lying on a plane surface. According to the anatomic region of interest, mild dorsiflexion during a volar examination or mild volar flexion during a dorsal examination may be required. ${ }^{5}$ Ulnar and radial deviations are useful when assessing radial and ulnar collateral ligaments, respectively. ${ }^{6}$ A large amount of gel and multiple-plane scans allow optimal visualization of the soft tissue structures of the wrist. ${ }^{5}$

\section{Normal Sonographic Appearance of Wrist Structures}

Tendons are composed of longitudinally oriented fascicles of type 1 collagen fibers. On sonograms, collagen bundles appear hyperechoic, resulting in a fibrillar pattern with multiple parallel lines in longitudinal planes and multiple dots in transverse planes. ${ }^{7}$ With the exception of the flexor carpi ulnaris and palmaris longus, all tendons of the wrist are enveloped by synovial sheaths that are sonographically depicted as thin echogenic fluid-containing structures. ${ }^{8}$ Some fluid may be present in small amounts in individuals with normal anatomy, but this fluid increases considerably if an inflammatory process occurs. ${ }^{9}$

Similarly to tendons, ligaments consist of type 1 collagen fibers, which are mainly oriented parallel to their long axis, forming bundles. Sonographically, they appear as fibrillar structures that lie deeper than wrist tendons. ${ }^{10}$ During sonographic evaluations of tendons and ligaments, the transducer needs to be held exactly orthogonal to the examined structure to avoid anisotropy, which is an artifactual decrease in echogenicity that mimics a pathologic change. Anisotropy appears to a lesser extent when depicting nerves. ${ }^{11}$

Peripheral nerves are composed of multiple axons or fibers surrounded by Schwann cells and a thin connective tissue, the endoneurium. Nerve fibers are grouped in fascicles enclosed within thin concentric layers of dense connective tissue, the perineurium, and assembled together in a matrix of loose connective tissue called the epineurium. Sonographically, in longitudinal planes, nerves show a fascicular pattern with multiple hypoechoic parallel lines comprising fascicles, separated by hyperechoic bands that correspond to the surrounding connective tissue; on transverse scans, nerves are depicted as hypoechoic round nerve fascicles surrounded by echogenic perineurium and epineurium. ${ }^{7}$

Blood vessels appear anechoic on sonograms. Veins are easily distinguished from arteries, as they collapse on application of moderate pressure from the transducer. Finally, sonography provides visualization of the skin, which is hyperechoic, the subcutaneous layer, which is hypoechoic with hyperechoic bands, and the cortical bone, which appears as a continuous hyperechoic line. ${ }^{8}$

\section{Extensor Tendons}

The extensor tendons cross the dorsum of the wrist through 6 osseofibrous tunnels or compartments (Figure 1), numbered from radial (1) to ulnar (6). ${ }^{12}$ The roof of these compartments is the extensor retinaculum, also known as dorsal carpal ligament, which is a focal thickening of the antebrachial fascia that spreads obliquely from the radius to the triquetrum and pisiform. Vertical fibrous septa extend downward from the retinaculum, forming the medial and lateral walls of the tunnels, and then attach to the radius, ulna, and distal radioulnar joint, which represent their floor. ${ }^{8,13}$ Regardless of the number of tendons it contains, each compartment is generally enclosed within a single synovial sheath. ${ }^{14}$

Figure 1. Schematic diagram of the extensor tendons of the wrist, superimposed on a wrist radiograph. The extensor tendons cross the dorsum of the wrist through 6 osseofibrous tunnels or compartments, numbered from radial $\left(1^{\circ}\right)$ to ulnar $\left(6^{\circ}\right)$. The extensor retinaculum forms the roof of these compartments and is represented as a gray band.

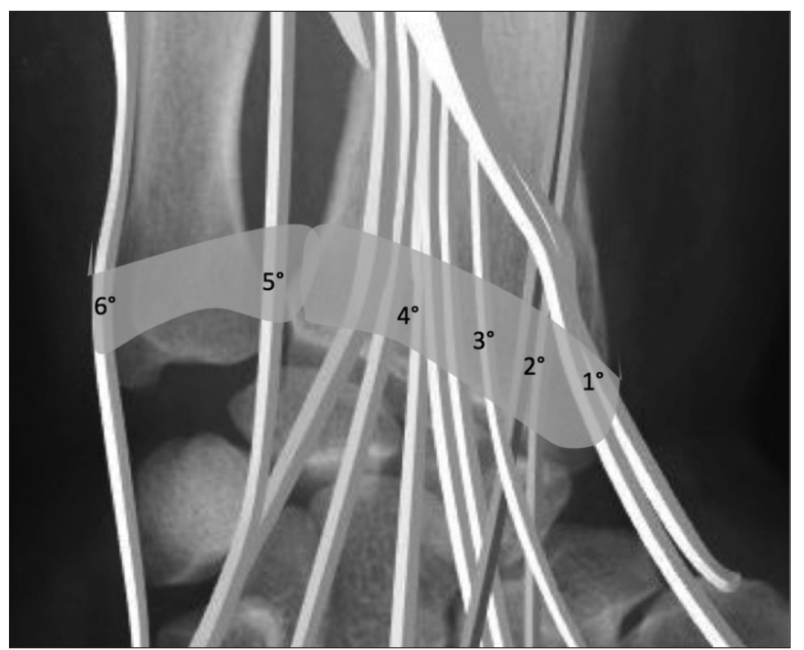


The first compartment (Figure 2A) is located on the radial aspect of the wrist and is occupied by the tendons of the abductor pollicis longus and extensor pollicis brevis muscles. ${ }^{11,13}$ An additional septum may separate the first compartment longitudinally into 2 parts, thereby determining an anatomic variation in which pathologic changes are confined to only 1 of these 2 tendons (commonly the extensor pollicis brevis tendon). The abductor longus tendon often has multiple terminal laminae instead of a single insertion. ${ }^{8}$ The second tunnel (Figure 2B) passes radially

Figure 2. Extensor compartments depicted on transverse scans. The abductor pollicis longus (APL) and extensor pollicis brevis (EPB) tendons lie within the first osteofibrous tunnel, on the radial side of the wrist (A). On the radial side of the Lister tubercle, the extensor carpi radialis longus (ECRL) and extensor carpi radialis brevis (ECRB) tendons run through the second osteofibrous tunnel (B). On the ulnar side of the Lister tubercle, the extensor pollicis longus (EPL) passes through the third osteofibrous tunnel (C). The extensor indicis proprius (EIP) and extensor digitorum communis (EDC) tendons lie within the fourth osteofibrous tunnel (D). At the level of the distal radioulnar joint, the extensor digiti minimi proprius (EDMP) tendon runs through the fifth osteofibrous tunnel (E). The extensor ulnaris carpi (ECU) tendon passes through the sixth osteofibrous tunnel, on the ulnar side of the wrist $(\mathbf{F})$. These tendons appear as fibrillar structures, and are encircled by the extensor retinaculum (arrows), which is hypoechoic because of anisotropy (A-F).
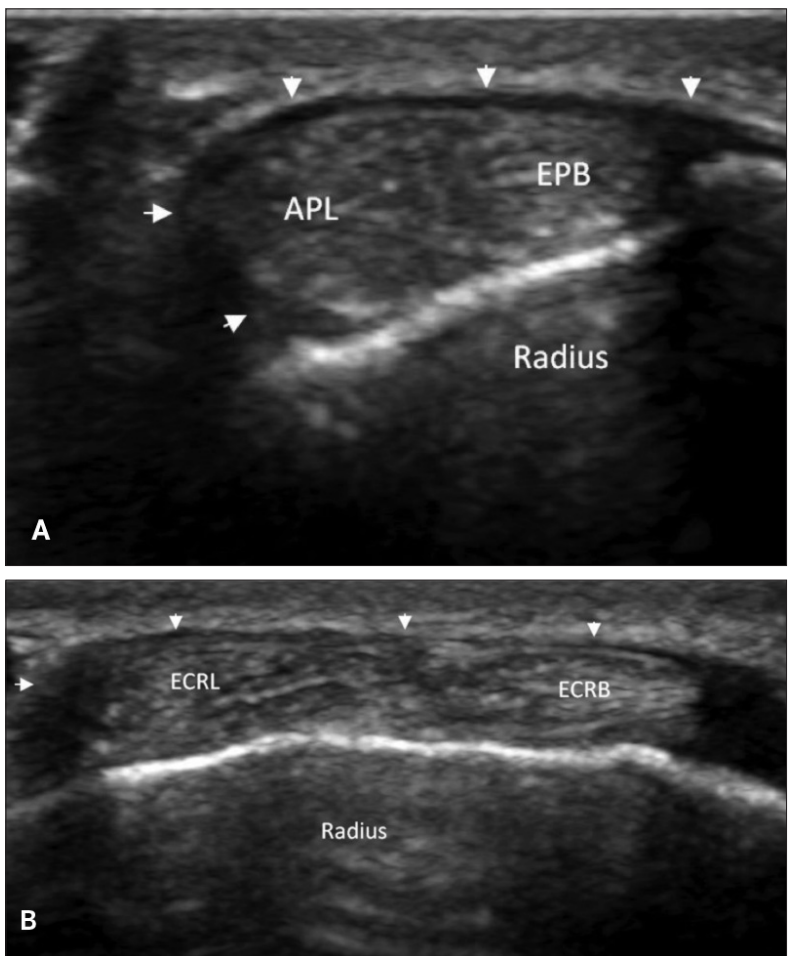

to the Lister tubercle, a dorsal bony prominence of the distal radius, and contains the tendons of the extensor carpi radialis longus and extensor carpi radialis brevis muscles; rare variants occur with accessory tendons and sheaths. ${ }^{11,13}$ Approximately 3.5 to $4.8 \mathrm{~cm}$ proximal to the Lister tubercle, the tendons of the second compartment run beneath those of the first compartment (Figure 3A), resulting in a friction point responsible for an overuse disorder known as proximal intersection syndrome. ${ }^{15,16}$ The extensor pollicis longus tendon runs through the third compartment (Figure 2C),
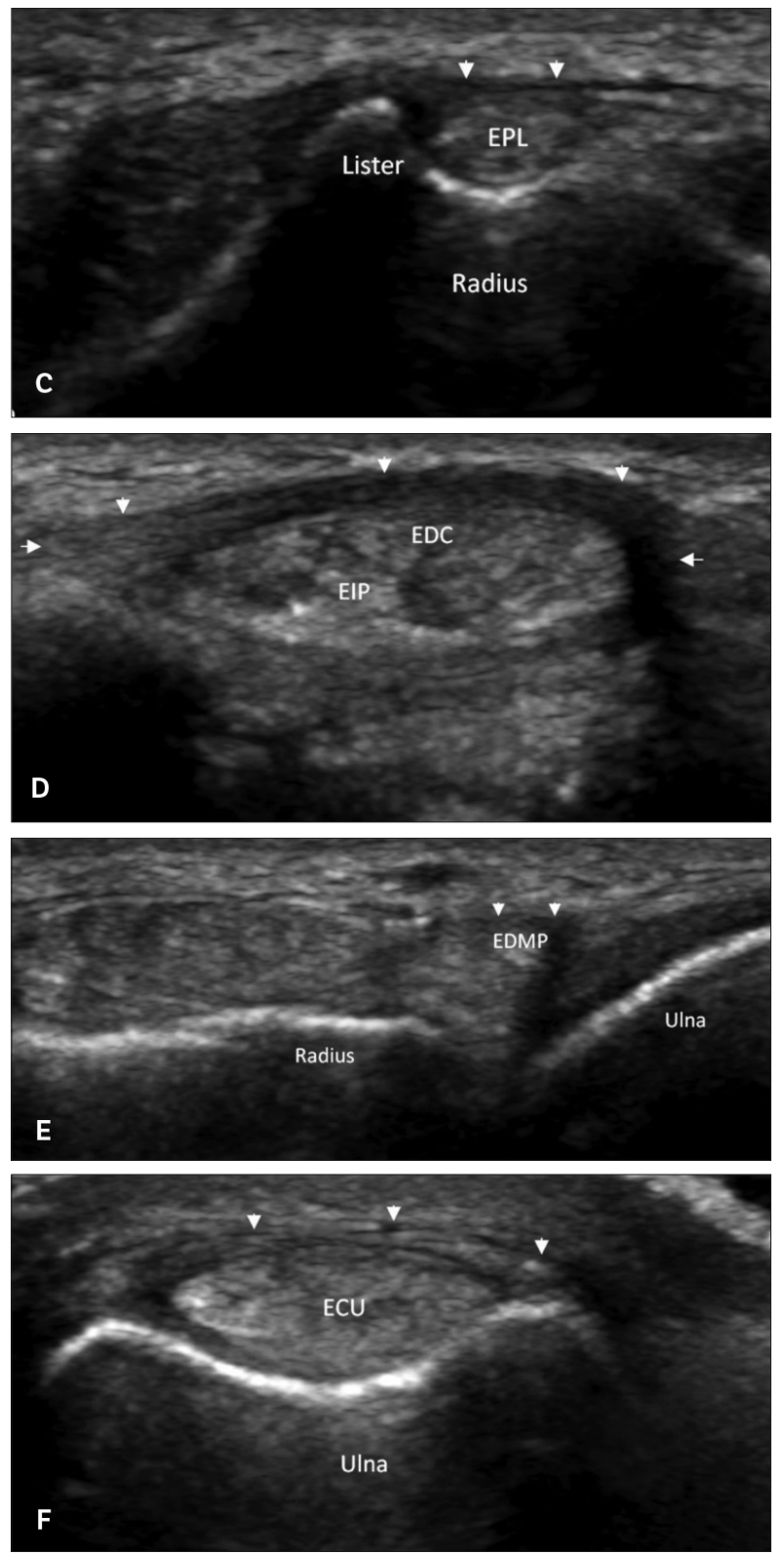
on the ulnar side of the Lister tubercle. ${ }^{11,13}$ It then proceeds over the tendons of the second compartment (Figure 3B), resulting in a friction point responsible for a further overuse disorder called distal intersection syndrome. ${ }^{16}$ The fourth tunnel (Figure 2D) is covered by the thickest portion of the extensor retinaculum and includes the tendons of the extensor digitorum communis and extensor indicis proprius muscles. ${ }^{11,13}$ The tendon of the extensor digiti minimi proprius muscle occupies the fifth tunnel (Figure 2E), which lies directly over the distal radioulnar joint. ${ }^{11,14}$ The sixth compartment (Figure 2F) is located at the ulnar aspect of the wrist and contains the tendon of the extensor carpi ulnaris muscle. ${ }^{11,13}$

\section{Flexor Tendons}

On the volar side of the wrist, there are 3 tendinous layers (Figure 4) that correspond to the muscle layers of the anterior forearm. The most superficial tendinous layer includes, from radial to ulnar, the tendons of the flexor carpi radialis, flexor palmaris longus, and flexor carpi ulnaris muscles.

Figure 3. Proximal and distal intersections. Proximal to the Lister tubercle, transverse scans depict the abductor pollicis longus (APL) and extensor pollicis brevis (EPB), which cross over the extensor carpi radialis longus (ECRL) and brevis (ECRB) tendons (A). During wrist movement, friction can develop between these groups of tendons, thus giving rise to proximal intersection syndrome. After passing through the third osteofibrous tunnel, the extensor pollicis longus (EPL) tendon passes over the ECRL and ECRB tendons, thus generating a further friction point called the distal intersection (B).
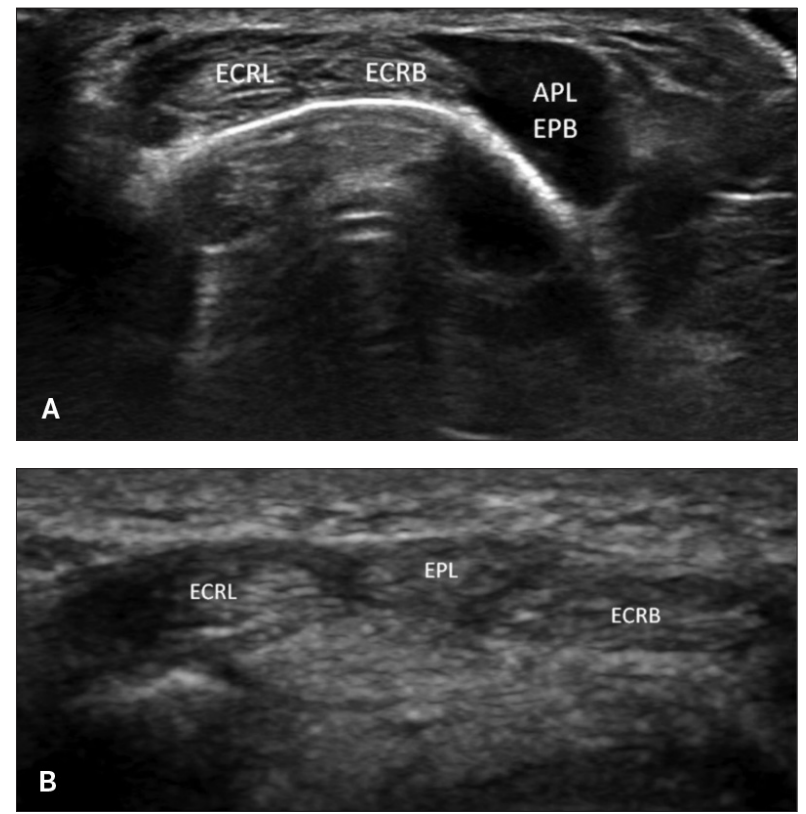

The tendon of the flexor carpi radialis (Figure 5) inserts with some fibers into the scaphoid and then proceeds distally. Unlike the other tendons of the superficial group, it is encircled within a synovial sheath that begins roughly $3 \mathrm{~cm}$ above the wrist. ${ }^{11,14}$ The palmaris longus is considered an accessory muscle in humans and is often used in tendon graft procedures. It is usually contiguous with the palmar fascia or inserts into the flexor retinaculum, also known as the transverse carpal ligament, a fibrous band that crosses the front of the carpus extending from the scaphoid and trapezium to the pisiform and hamate. The flexor carpi ulnaris tendon (Figure 6) inserts into the pisiform, the flexor retinaculum, and the hook of the hamate and also proceeds distally with a few fibers. ${ }^{8}$ Sometimes, it is separated from the pisiform by a bursa, which is sonographically recognizable only if it contains fluid. ${ }^{17}$

The second layer contains the tendons of the flexor digitorum superficialis muscle, and the third layer includes the tendons of the flexor digitorum profundus and flexor pollicis longus. The tendons of the flexor digitorum superficialis and profundus muscles (4 tendons in each), the flexor pollicis longus tendon, and the median nerve all pass through the carpal tunnel (Figure 5). The carpal tunnel is delimited dorsally by the carpal bones (floor) and volarly by the flexor retinaculum (roof). It is conventionally divided into 2 parts: a proximal segment whose walls are the pisiform on the ulnar side and the scaphoid on the radial side,

Figure 4. Schematic diagram of the flexor tendons of the wrist. 1 indicates flexor digitorum superficialis tendons; 2 , flexor digitorum profundus tendons; 3 , flexor pollicis longus tendon; 4, flexor carpi radialis tendon; 5, palmaris longus tendon; and 6, flexor carpi ulnaris tendon. The flexor retinaculum is represented as a gray band that crosses the front of the carpus and forms the roof of the carpal tunnel.

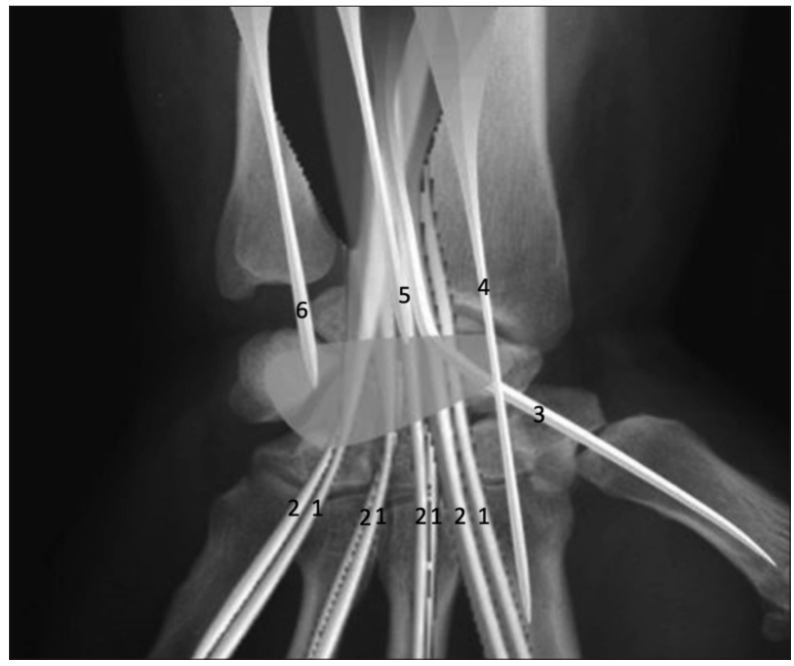


and a distal segment delimited by the hook of the hamate on the ulnar side and the tubercle of the trapezium on the radial side. The flexor pollicis longus tendon, located at the radial border of the carpal tunnel, has its own sheath. The tendons of the flexors digitorum superficialis and profundus overlap on the ulnar side of the tunnel and share a common sheath. ${ }^{8,18}$ The Guyon canal (Figure 6) is superficial to the carpal tunnel and extends from the pisiform to the hook of the hamate. It is delimited dorsally by the flexor retinaculum (floor) and volarly by the palmar carpal ligament, which is the distal extent of the antebrachial fascia and the palmaris brevis muscle (roof). The ulnar nerve and ulnar artery pass through this canal, and 1 or 2 veins may be present. ${ }^{19,20}$ The fourth muscle layer of the anterior forearm is represented by the pronator quadratus, which originates and inserts within the forearm and does not extend to the wrist. ${ }^{12}$

Figure 5. Flexor carpi radialis tendon and carpal tunnel. During a volar examination of the wrist, a transverse scan depicts the flexor pollicis longus (FPL) tendon, the flexor digitorum superficialis (FDS) and flexor digitorum profundus (FDP) tendons, as well as the median nerve (MN), all running through the carpal tunnel. Tendons appear as fibrillar structures, and the median nerve shows a fascicular pattern. The flexor retinaculum (arrows) circumscribes the carpal tunnel superiorly and is hypoechoic because of anisotropy. Right above the carpal tunnel, the flexor carpi radialis (FCR) tendon and ulnar artery (UA) are visualized.

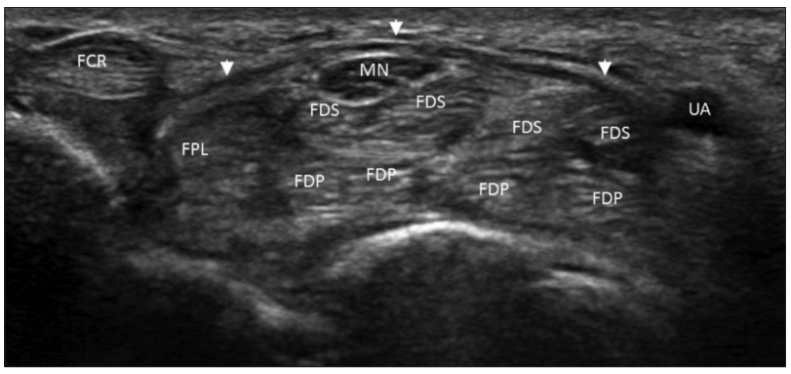

Figure 6.Guyon canal and flexor carpi ulnaris tendon. During a volar examination of the wrist, a transverse scan shows the ulnar artery (UA) and ulnar nerve $(U N)$ within the Guyon canal. The ulnar nerve appears as a fascicular structure, and it has already divided into its superficial and deep branches. The ulnar artery is anechoic. On the ulnar side, the Guyon canal is delimited by the pisiform bone and flexor carpi ulnaris (FCU) tendon.

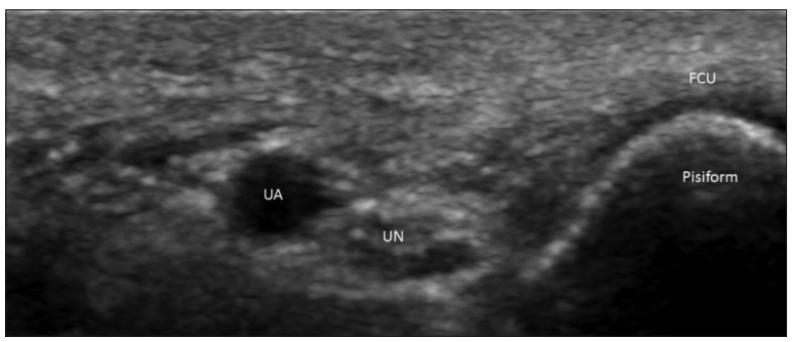

\section{Common Variations in Muscles and Tendons}

The forearm and hand muscles show several anatomic variants at the wrist. They are most frequently asymptomatic and represent incidental findings but may also present as palpable swellings or generate nerve compression within the carpal tunnel or Guyon canal. ${ }^{19}$ An accessory extensor carpi radialis muscle may be found between the extensor carpi radialis brevis and longus and originates directly (properly the accessory extensor carpi radialis) or independently (intermediate extensor carpi radialis) from the main muscles. Its tendon runs through the second extensor compartment of the wrist or, rarely, through a separated fascial tunnel beneath the extensor retinaculum (if the accessory muscle arises from the extensor carpi radialis longus). ${ }^{21}$ The prevalence of an additional muscle amounts to $10 \%$ to $24 \%$ of the population in cadaveric studies, with frequent bilaterality. ${ }^{22,23}$ The palmaris longus is also highly variable. Its agenesis may be unilateral or bilateral and occurs in $4 \%$ to $25 \%$ of individuals, with a higher prevalence in white populations. All other palmaris longus variants are observed in approximately $9 \%$ of the population and mainly include a duplicated, digastric, entirely muscular, and reversed palmaris longus (Figure 7), a structure that is tendinous proximally and muscular distally (in contrast to the normal palmaris longus). ${ }^{24,25}$ Among the accessory hypothenar muscles, the accessory abductor digiti minimi (Figure 8 ) is the most common and occurs in $24 \%$ of individuals. It arises from the antebrachial fascia or, alternatively, from the palmaris longus tendon in the distal forearm before coursing through the Guyon canal in a superficial position. ${ }^{26}$ On the dorsum of the wrist, an extensor digitorum brevis manus muscle is described in $1.6 \%$ of the population, most frequently originating from the dorsal capsule deeper than the extensor retinaculum. ${ }^{27}$ Other rare anatomic variants at the wrist are the accessory flexor digitorum superficialis indicis muscle, which is characterized by a muscle belly replacing the tendon directed to the index finger, and the flexor carpi radialis brevis vel profundus muscle, which arises from the distal radius and runs deeper than the flexor carpi radialis. ${ }^{21}$

\section{Nerves}

The sensory branch of the radial nerve, the ulnar nerve, and the median nerve cross the wrist. The sensory branch of the radial nerve (Figure 9) is superficial and divides into its terminal cutaneous branches after passing over the myotendinous junctions of the abductor pollicis longus and the extensor pollicis brevis. ${ }^{8}$ 
The median nerve (Figure 5) runs through the carpal tunnel immediately beneath the flexor retinaculum, above the flexor pollicis longus tendon and the laminae of the flexor digitorum superficialis, and gives rise to its terminal

Figure 7. Reversed palmaris longus. On the volar surface of the forearm, transverse scans show the distal belly of the reversed palmaris longus (RPL) lying in a superficial position, adjacent to the flexor carpi radialis (FCR) tendon. The median nerve (arrow) is deeper than the RPL and FCR (A). The muscular belly crosses the carpal tunnel right above the median nerve (B, arrow).
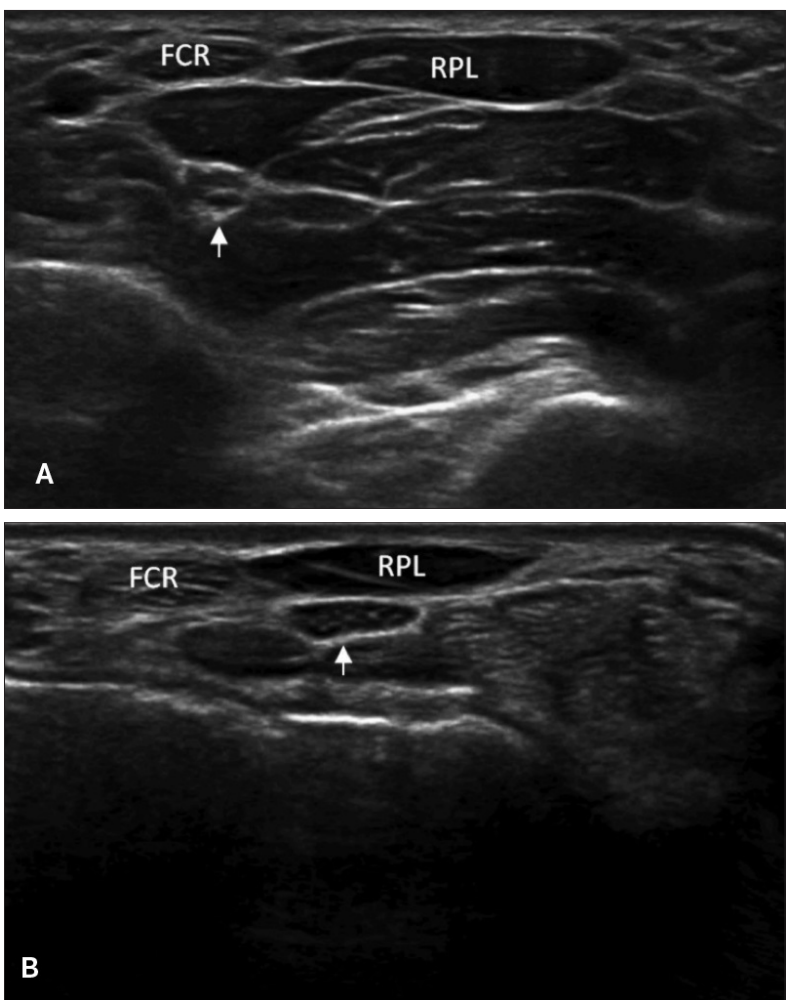

Figure 8. Accessory abductor digiti minimi muscle. On the ulnar side of the wrist, a transverse scan may depict an accessory abductor digiti minimi (AADM) muscle within the Guyon canal. The ulnar artery (UA) and the superficial and deep branches (arrows) of the ulnar nerve are also visualized.

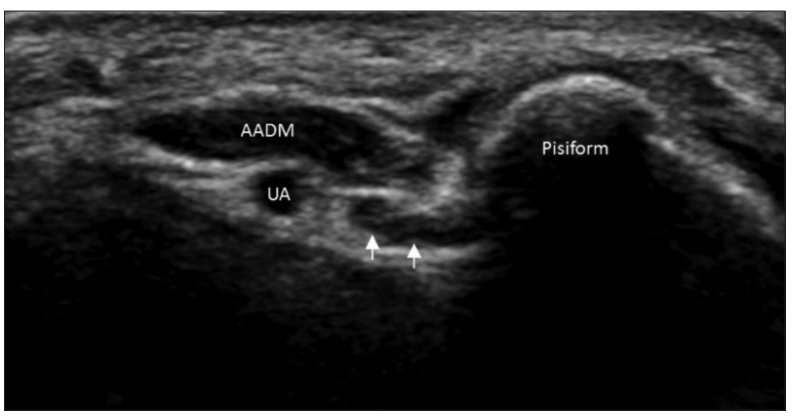

branches after emerging from the tunnel. ${ }^{11}$ The small palmar cutaneous branch of the median nerve (Figure 10) arises just proximal to the carpal tunnel and pierces the antebrachial fascia before innervating the cutaneous and subcutaneous tissues of the palm. In some individuals, its course is extremely superficial, so this configuration should be examined closely if a surgical incision of the flexor reti-

Figure 9. Superficial branch of the radial nerve. On the dorsal side of the distal forearm, transverse scans show the superficial branch of the radial nerve (arrow), appearing as a fascicular structure that passes radial to the extensor carpi radialis longus (ECRL) and brevis (ECRB) tendons in a superficial position (A). At the level of the proximal intersection, it crosses over the myotendinous junction of the abductor pollicis longus $(\mathrm{APL})$ and extensor pollicis brevis (EPB; $\mathbf{B}$ ) and then becomes ulnar (C).
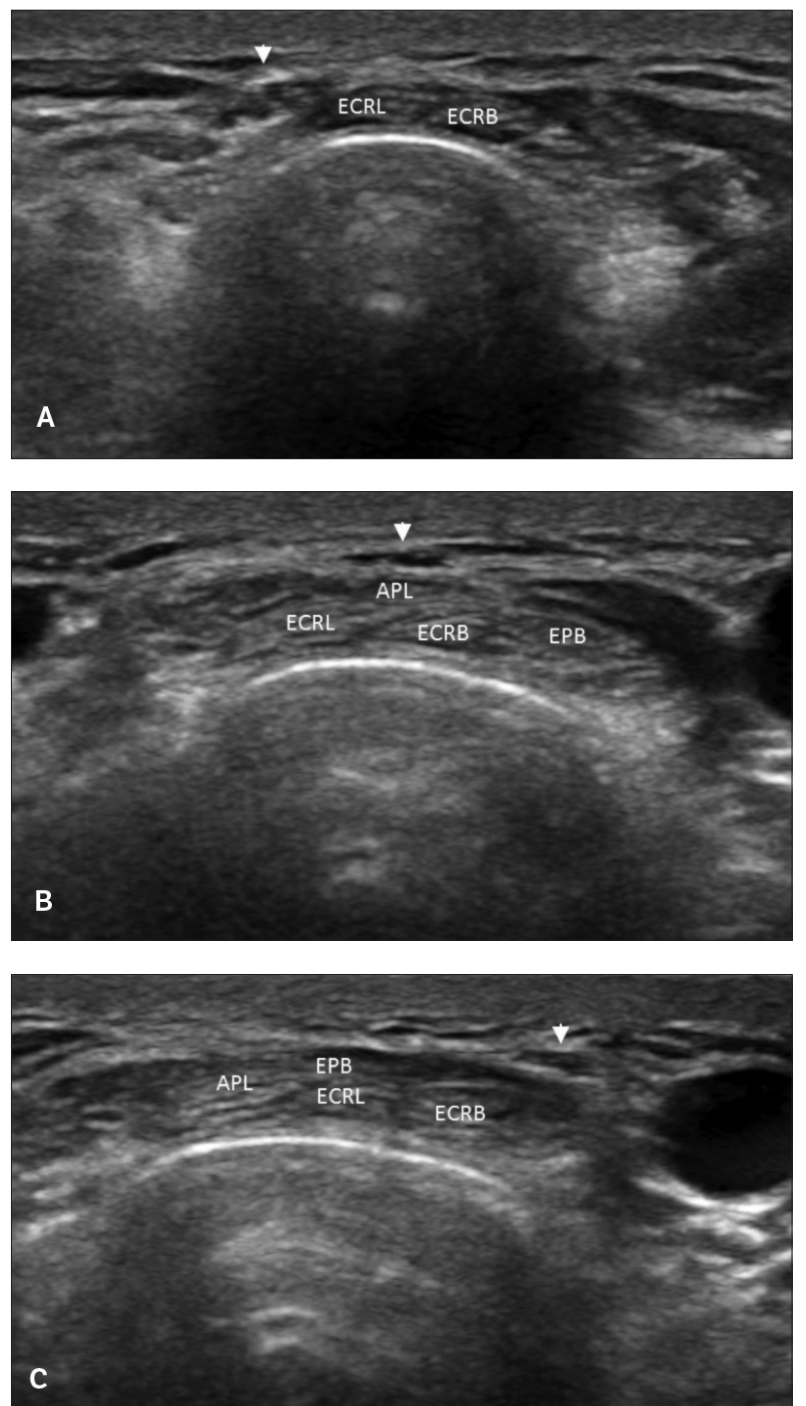
naculum is planned..$^{8,12}$ One important anatomic variation is a bifid median nerve (Figure 11), which occurs in roughly $20 \%$ of individuals and is often associated with a persistent median artery (Figure 12). The median artery arises from the ulnar artery and passes along the ulnar side of the median nerve or, if the median nerve is bifid, generally between the 2 median nerves. ${ }^{28}$
The ulnar nerve (Figure 6) usually enters the Guyon canal after dividing into its superficial sensory and deep motor branches, but in around $10 \%$ of individuals, this bifurcation takes place inside the tunnel. The ulnar artery passes along the radial side of the ulnar nerve but is also variable, as its deep palmar branch may pass over, between, or beneath the nerve terminal branches. 8,20

Figure 10. Small cutaneous branch of the median nerve. The small cutaneous branch (arrows) of the median nerve (MN) is depicted on transverse scans at the level of the wrist, where it arises from the median nerve $(\mathbf{A})$ and pierces the flexor retinaculum (B).
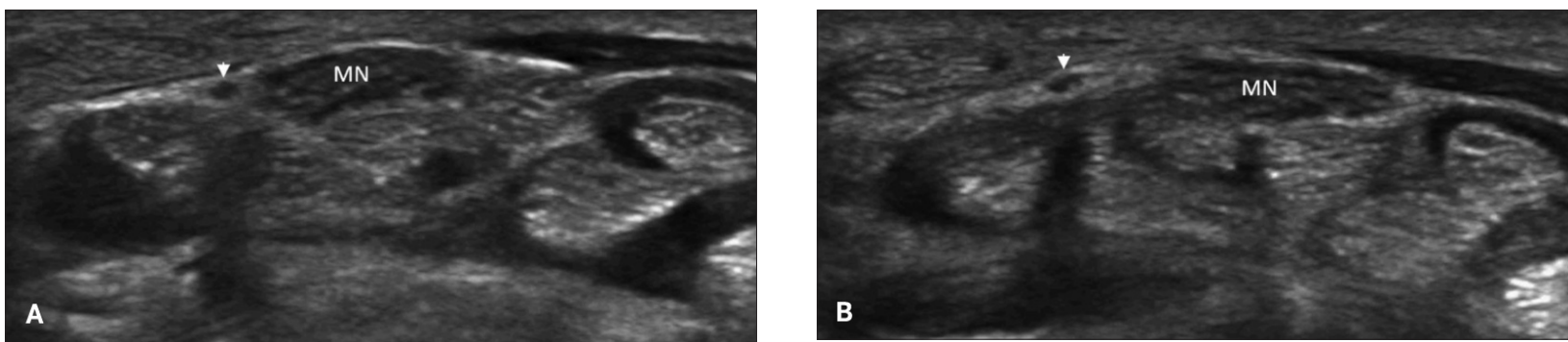

Figure 11. Bifid median nerve. On the volar surface of the middle forearm, transverse scans show a single median nerve (arrows) lying between the flexor digitorum superficialis and profundus muscles $(\mathbf{A})$. When proceeding distally, the main nerve trunk bifurcates into 2 branches (B), which are close to each other at the level of the pronator quadratus (PQ) muscle (C). Within the carpal tunnel, 2 median nerves can be seen in the usual position, right beneath the flexor retinaculum (D).
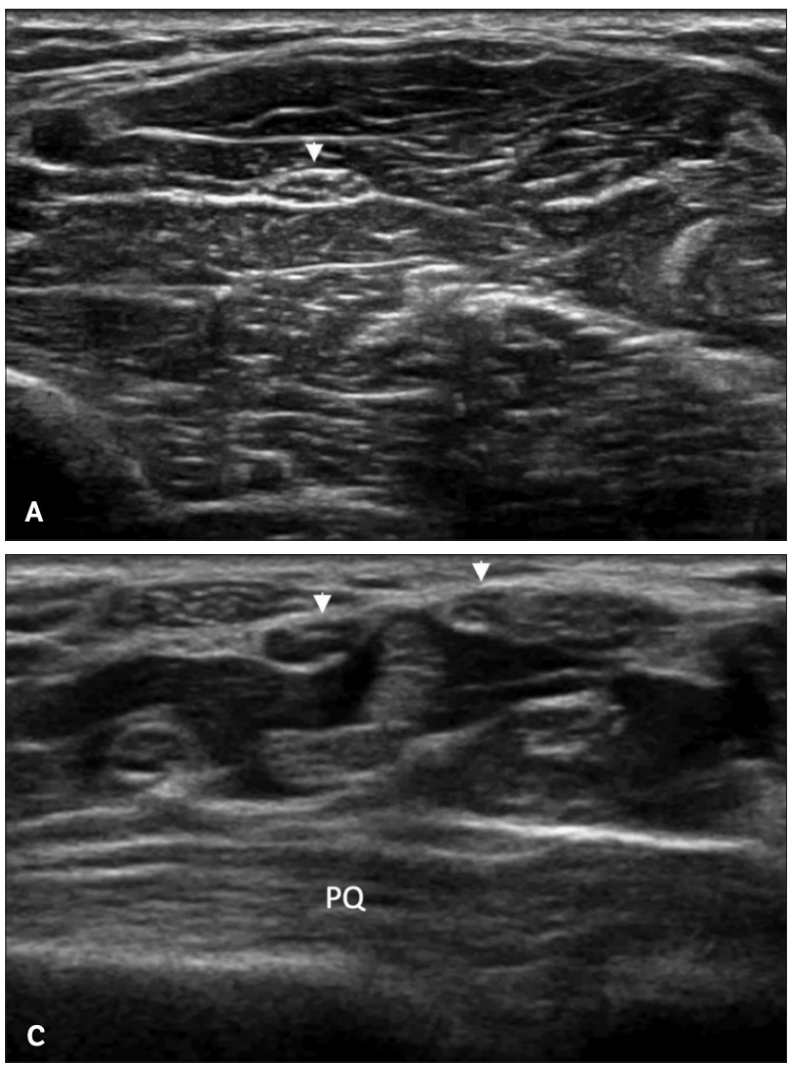
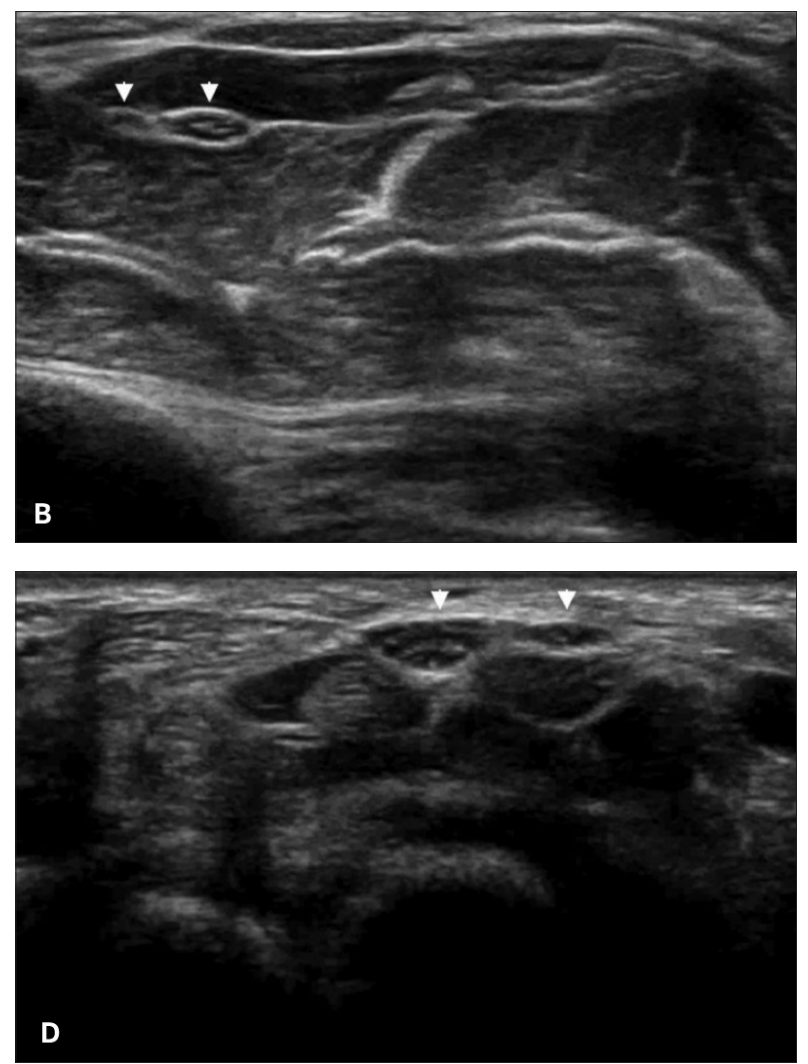


\section{Extracapsular Ligaments}

The extensor and flexor retinacula, previously referred to as the dorsal and transverse carpal ligaments, are extracapsular, as they lie superficial to the wrist fibrous capsule. The pisotriquetral ligament belongs to the same group and connects the triquetrum and pisiform. ${ }^{12}$

\section{Intracapsular Extrinsic Ligaments and Tri- angular Fibrocartilage Complex}

The intracapsular ligaments are located between the synovial and fibrous layers of the wrist joint and interdigitate with each another, leading to an unclear demarcation of their fibers. ${ }^{12}$ They are named for the origin and insertion bones, proximal to distal and radial to ulnar, and classified into extrinsic and intrinsic ligaments. ${ }^{29}$

Figure 12. Persistent median artery and bifid median nerve. In the distal forearm, transverse scans show the median artery (MA) running ulnar to 2 median nerves (A, arrows). At the level of the wrist, it becomes more superficial and crosses the carpal tunnel between the 2 nerve trunks, right below the flexor retinaculum (B)
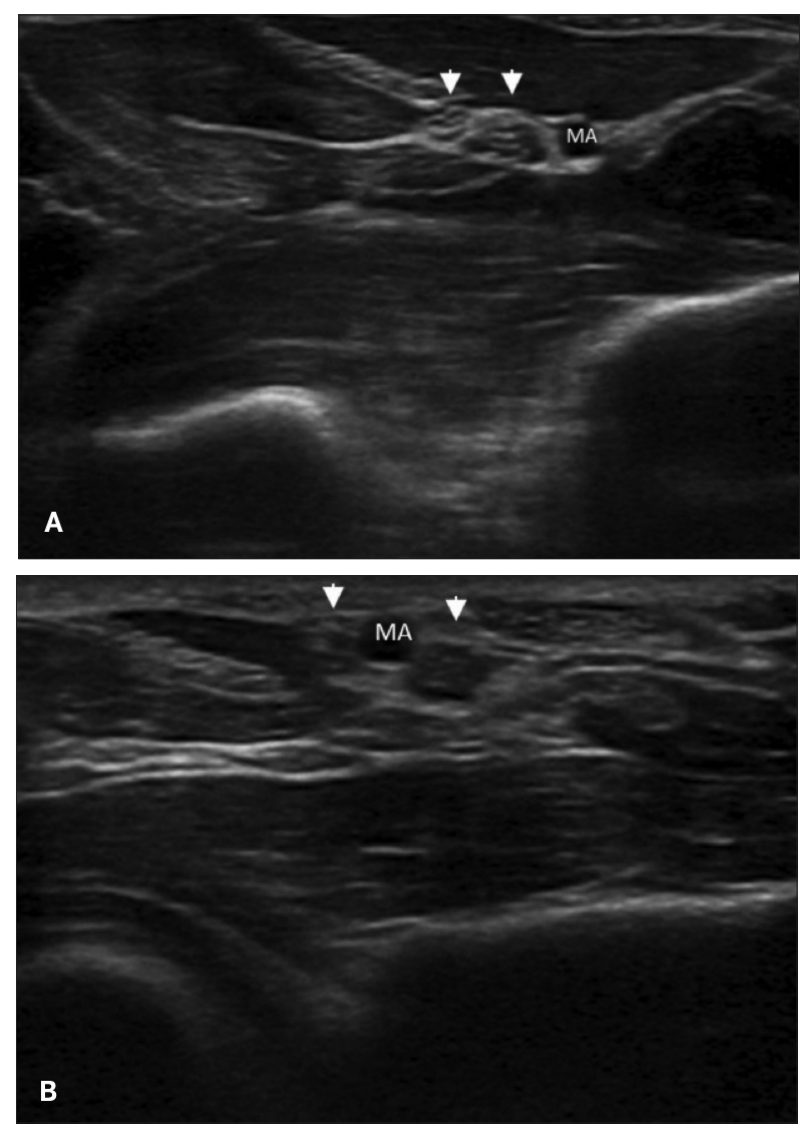

The extrinsic ligaments connect the carpus with the forearm bones or the radioulnar ligaments and are further subdivided into dorsal, palmar, and collateral ligaments. ${ }^{30-32}$ The extrinsic palmar ligaments (Figure 13) form 2 arcuate structures, respectively attaching to the capitate on the distal carpal row (greater arc) and outlining the lunate on the proximal row (lesser arc). ${ }^{6}$ The triangular area between these arcs is called the space of Poirier and is vulnerable to injury and dislocation, as it is not covered by any ligaments. ${ }^{33}$ The radioscaphocapitate ligament (Figure 14) originates at the distal radius, from the radial styloid to approximately the middle of the scaphoid fossa, and inserts into the scaphoid and capitate. The long radiolunate ligament (Figure 15), also known as the palmar radiolunotriquetral or radiotriquetral ligament, connects the remaining part of the scaphoid fossa with the lunate and triquetrum. The short radiolunate ligament (Figure 16) extends from the lunate fossa of the radius to the lunate. ${ }^{30,34}$ The radio-

Figure 13. Schematic diagram of the palmar extrinsic ligaments of the wrist, superimposed on a wrist radiograph. 1 indicates radioscaphocapitate ligament; 2 , long radiolunate ligament; 3 , short radiolunate ligament; 4, ulnolunate ligament; 5, ulnotriquetral ligament; and 6, ulnocapitate ligament.

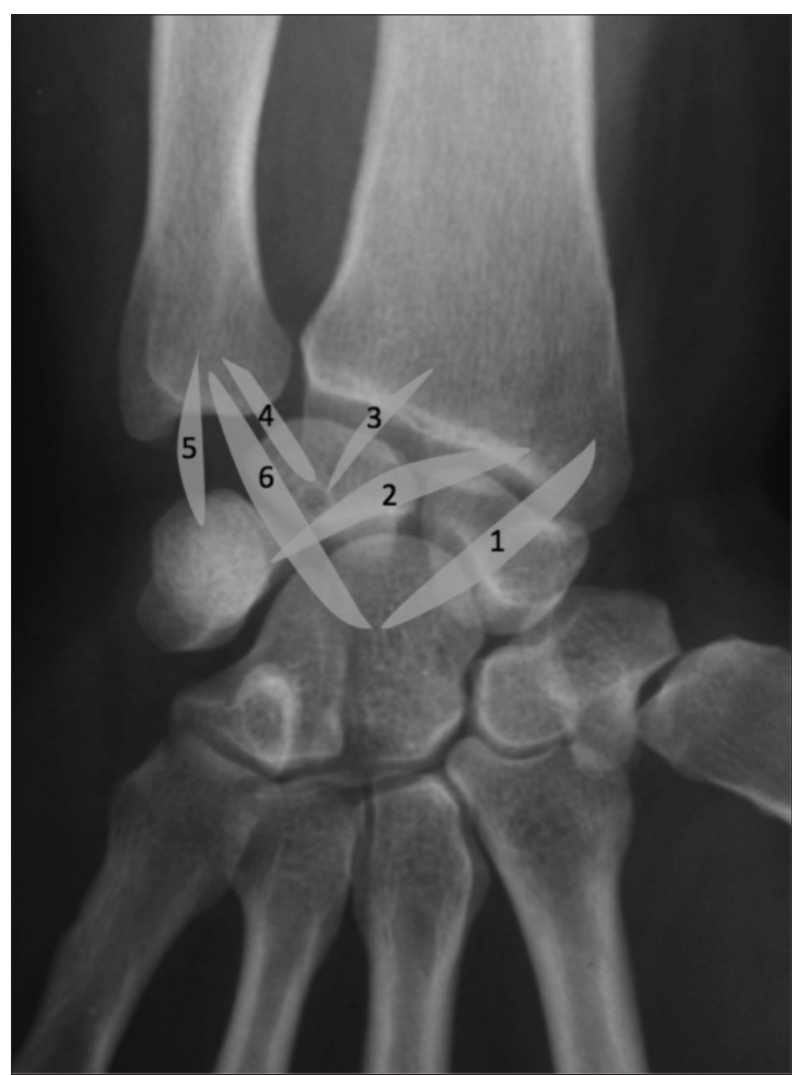


scapholunate ligament (Figure 17), also known as the ligament of Testut and Kuenz, lies between the long and short radiolunate ligaments. It is not a true ligament in the histologic sense but, rather, a neurovascular structure with vascular origins from the anterior interosseous and radial arteries and a neural origin from the anterior interosseous nerve. ${ }^{35}$ The palmar ulnolunate and palmar ulnotriquetral ligaments (Figures 18 and 19) originate from the volar radioulnar ligament, with no clear proximal demarcation, and attach to the lunate and triquetrum, respectively. The palmar ulnocapitate ligament (Figure 20) originates from the ulnar fovea and extends distally superficial to the palmar ulnolunate and ulnotriquetral ligaments. It terminates on the capitate, where it interdigitates with the radioscaphocapitate ligament, forming the arcuate ligament. The arcuate ligament corresponds to the greater arc previously described. ${ }^{30,34}$

Figure 14. Radioscaphocapitate ligament. The transducer is placed in the longitudinal plane at the palmar aspect of the distal radius and then moved distally and slightly rotated toward the capitate. The ligament (arrows) is visualized along its long axis as a fibrillar structure that originates from the distal radius and attaches to the scaphoid and capitate.

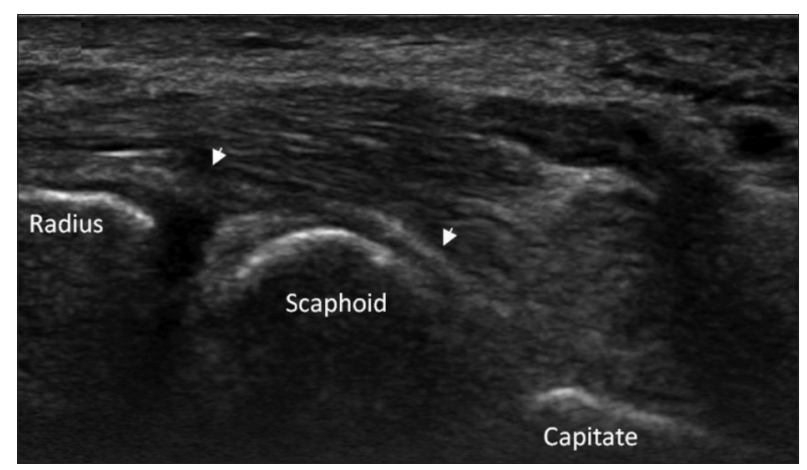

Figure 15. Long radiolunate ligament. The transducer is placed in the longitudinal plane at the palmar aspect of the distal radius; thereafter, it is moved distally and rotated toward the second and third bones of the first carpal row. The ligament (arrows) is visualized along its long axis as a fibrillar structure that originates from the distal radius and inserts into the lunate and triquetrum.

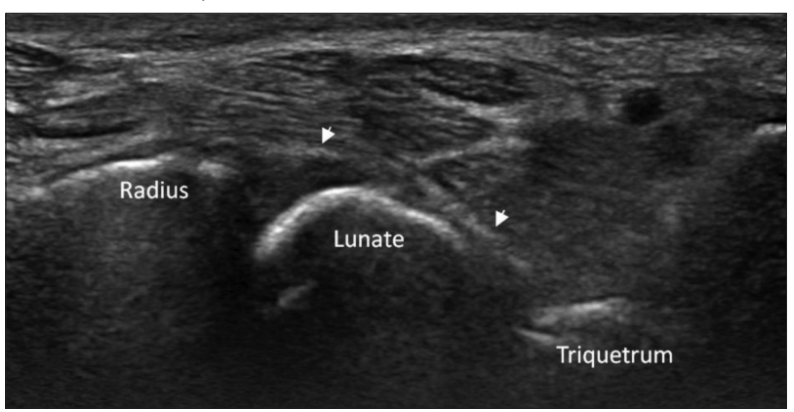

Figure 16. Short radiolunate ligament. The transducer is placed in the longitudinal plane at the palmar aspect of the distal radius and then moved distally and slightly rotated toward the lunate. The ligament (arrows) is visualized along its long axis as a fibrillar structure extending from the distal radius to the lunate.

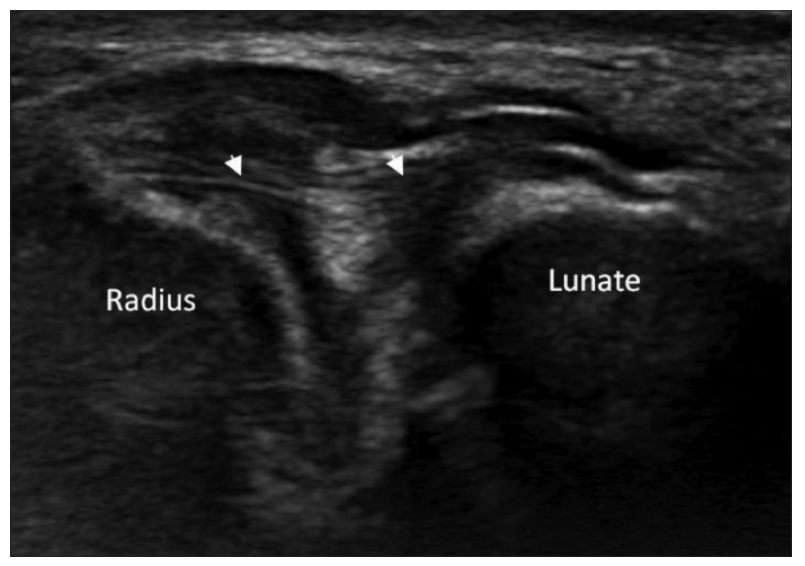

Figure 17. Radioscapholunate ligament. The radioscapholunate ligament is not a true ligament in the histologic sense but, rather, a neurovascular structure. Its neural component arises from the anterior interosseus nerve (arrow), which is visualized on transverse scans at the level of pronator quadratus (PQ) muscle, where it originates from the median nerve right beneath the flexor carpi radialis (FCR) tendon (A). The transducer is then moved distally and slightly rotated toward the lunate. The ligament (arrows) shows a heterogeneous appearance and passes over the scapholunate joint. Its identification is also facilitated by color Doppler imaging (B).
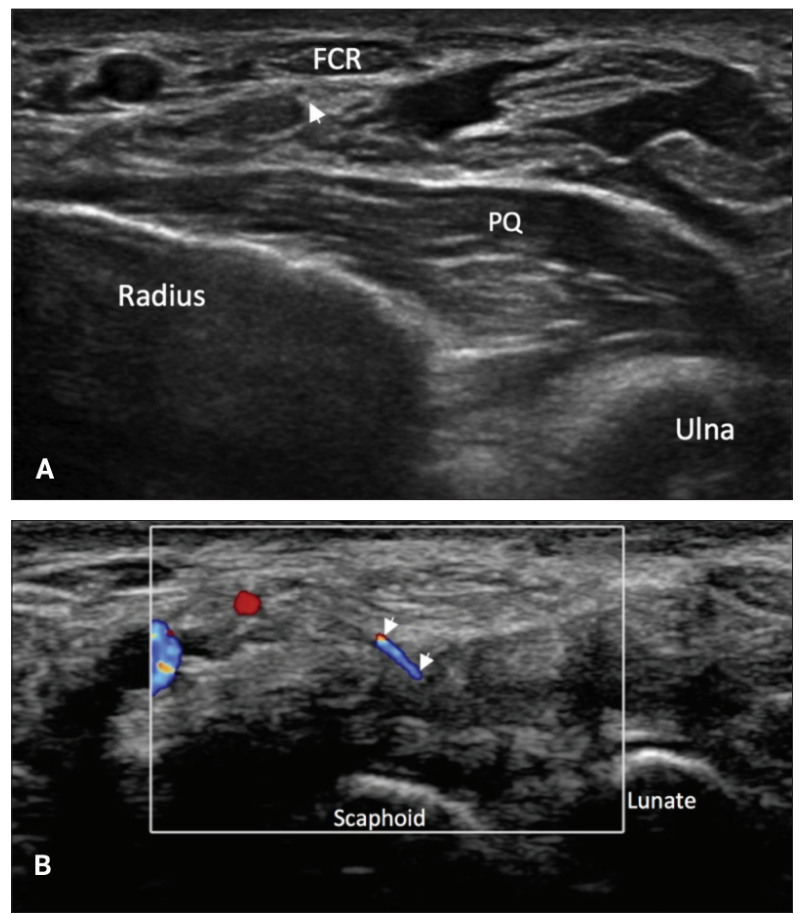
The extrinsic dorsal and collateral ligaments (Figure 21) are biomechanically less important than the palmar ones. ${ }^{36}$ The dorsal radiocarpal ligament (Figure 22), also known as the dorsal radiotriquetral or dorsal radiolunotriquetral ligament, originates distally to the Lister tubercle, attaches with

Figure 18. Ulnolunate ligament. The transducer is placed in the longitudinal plane at the palmar aspect of the distal ulna and then moved distally and slightly rotated toward the lunate. The ligament (arrows) is visualized along its long axis as a fibrillar structure arising from the distal ulna (properly from the volar radioulnar ligament) to the lunate.

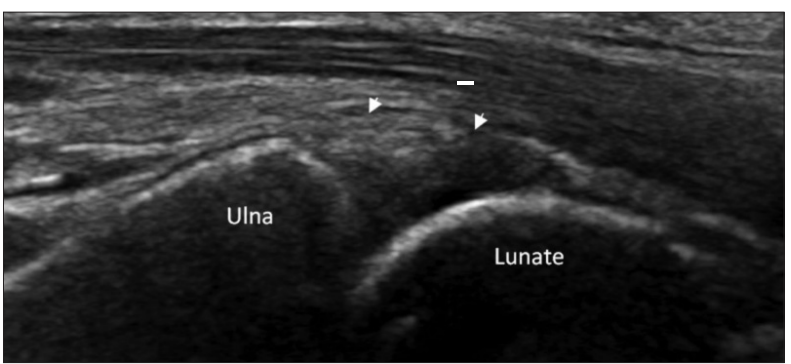

Figure 19. Ulnotriquetral ligament. The transducer is placed in the longitudinal plane at the palmar aspect of the distal ulna and then moved distally. The ligament (arrows) is visualized along its long axis as a fibrillar structure extending from the distal ulna (properly from the volar radioulnar ligament) to the triquetrum.

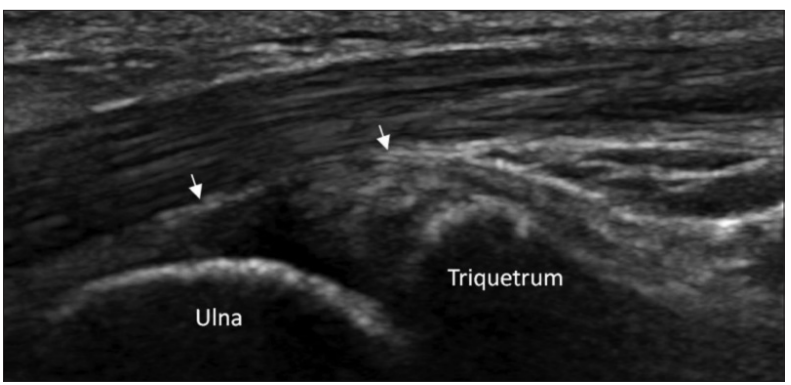

Figure 20. Ulnocapitate ligament. The transducer is placed in the longitudinal plane at the palmar aspect of the distal ulna, and then moved distally and slightly rotated toward the capitate. The ligament (arrows) is visualized along its long axis as a fibrillar structure that originates from the distal ulna, courses over the lunotriquetral joint, and terminates on the capitate.

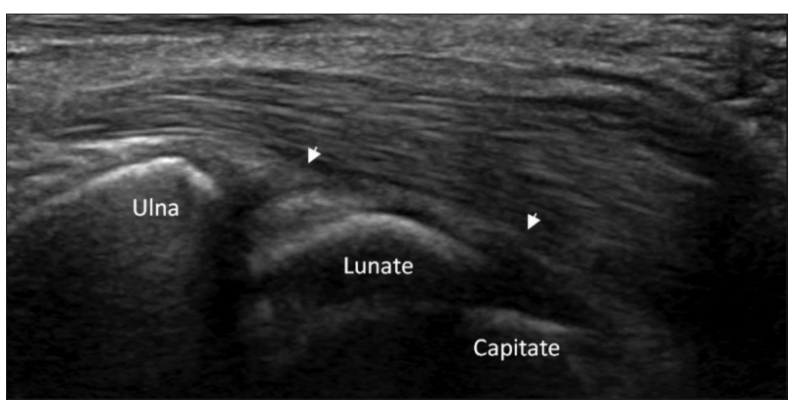

some fibers to the lunate, and terminates on the triquetrum. The radial collateral ligament (Figure 23) is deeper than the first extensor compartment and connects the radial styloid with the scaphoid. The ulnar collateral ligament (Figure 24) is deeper than the sixth extensor compartment and extends

Figure 21. Schematic diagram of the dorsal and collateral extrinsic ligaments of the wrist, superimposed on a wrist radiograph. 7 indicates dorsal radiocarpal ligament; 8 , radial collateral ligament; and 9, ulnar collateral ligament.

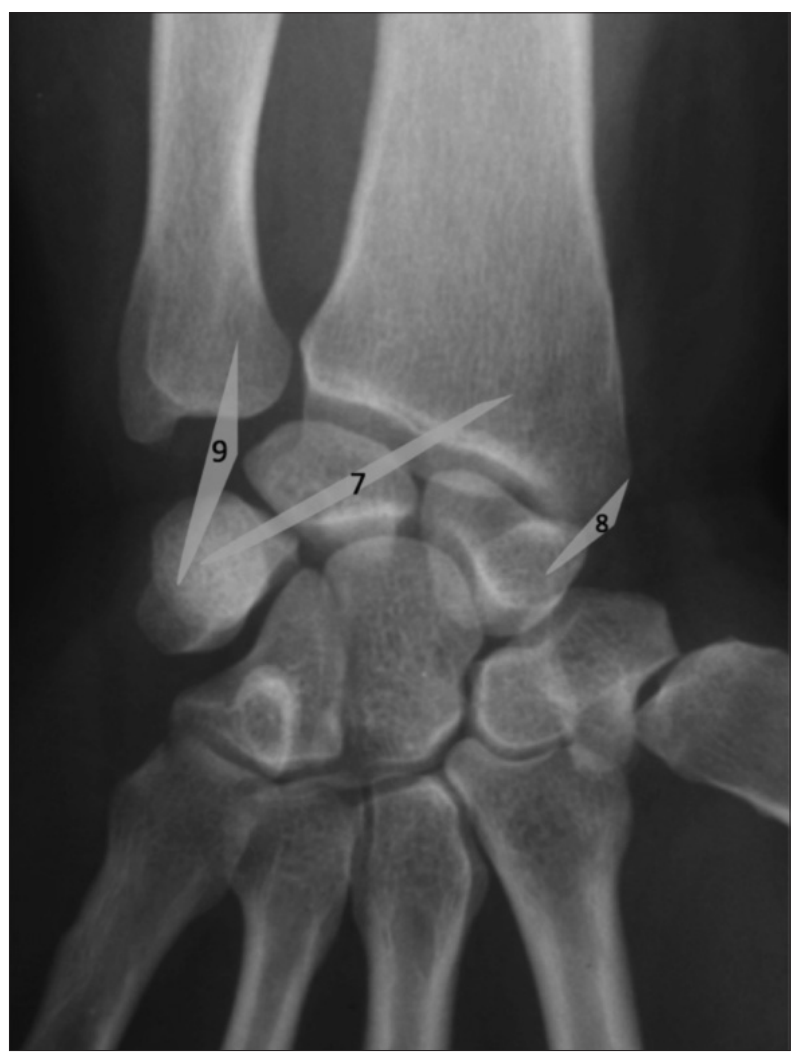

Figure 22. Dorsal radiocarpal ligament. The transducer is placed in the transverse plane at the dorsal radius, over the Lister tubercle, and then moved distally and slightly rotated toward the second and third bones of the first carpal row. The ligament (arrows) is visualized along its long axis as a fibrillar structure that originates from the dorsal radius, distally to the Lister tubercle, and inserts into the lunate and triquetrum. It is deeper than the fourth extensor compartment.

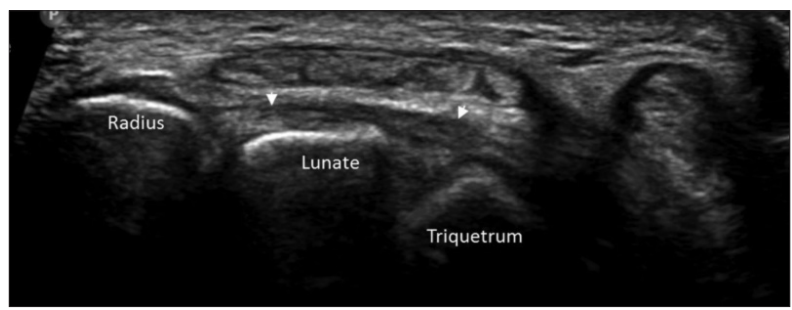


from the ulnar styloid to the triquetrum. ${ }^{30,34}$ Together with the triangular fibrocartilage, meniscus homologue, palmar and dorsal radioulnar ligaments, and extensor carpi ulnaris tendon sheath, it forms the triangular fibrocartilage complex (Figures 25-27), which confers stability to the ulnar wrist.

Figure 23. Radial collateral ligament. The transducer is placed in the longitudinal plane at the distal radius, over the styloid process, and then moved distally. The ligament (arrows) is visualized along its long axis as a fibrillar structure extending from the radial styloid process to the scaphoid.

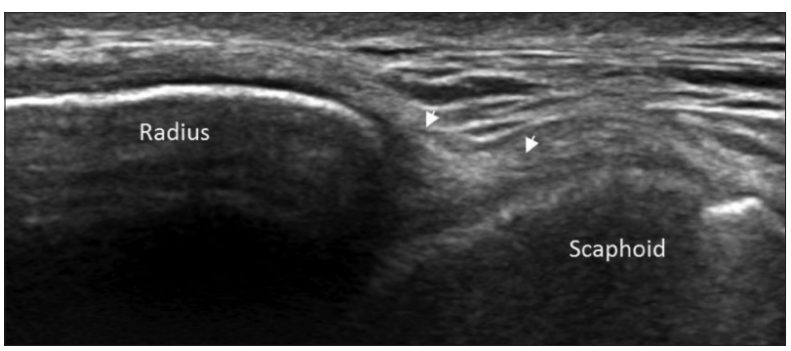

Figure 24. Ulnar collateral ligament. The transducer is placed in the longitudinal plane at the distal ulna, over the styloid process, and then moved distally. What is considered the ulnar collateral ligament (arrows) does not show a fibrillar pattern but is an echogenic joint capsule thickening that extends from the ulnar styloid process to the triquetrum. It is deeper than the extensor carpi ulnaris tendon (ECU).

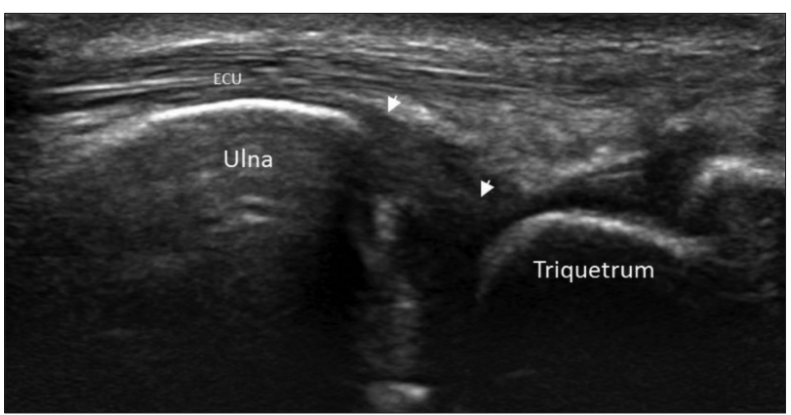

Figure 25. Triangular fibrocartilage complex (dorsal aspect). The transducer is placed in the longitudinal plane at the distal ulna and then moved distally. The triangular fibrocartilage (arrows) is visualized as an echogenic structure attached to the distal aspect of the ulna. The meniscus homologue $(\mathrm{MH})$ also appears echogenic and is adjacent to the triangular fibrocartilage disk. The extensor carpi ulnaris tendon (ECU) lies superficial to these structures.

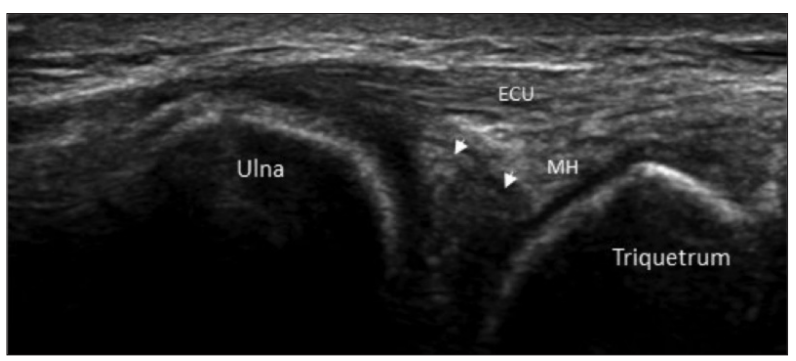

The triangular fibrocartilage is a biconcave disk between the radius (base) and ulnar styloid (apex). The meniscus homologue is an embryologic remnant of the ulnar wrist that is only occasionally present. The palmar and dorsal radioulnar ligaments extend on the anterior and posterior surfaces of the distal radioulnar joint, respectively. ${ }^{12,37}$

Figure 26. Volar radioulnar ligament. The transducer is placed in the transverse plane at the volar aspect of the distal forearm. The ligament (arrows) is visualized along its long axis as a fibrillar structure extending between the distal radius and the distal ulna. The triangular fibrocartilage (TFC) is also depicted.

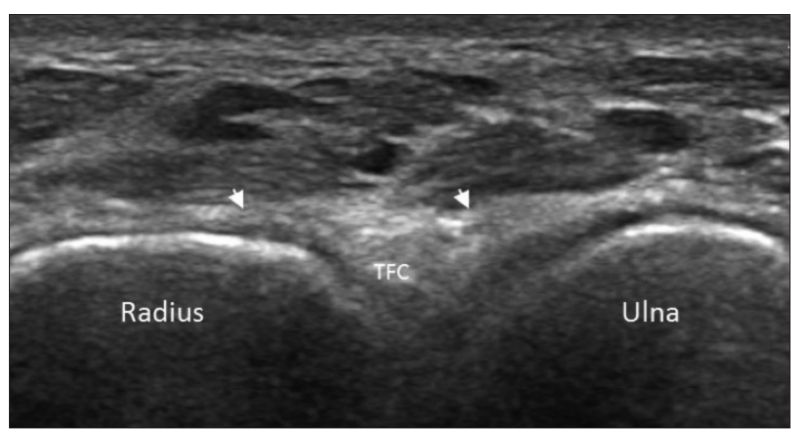

Figure 27. Dorsal radioulnar ligament. The transducer is placed in the transverse plane at the dorsal aspect of the distal forearm. The ligament (arrows) is visualized along its long axis as a fibrillar structure extending between the distal radius and the distal ulna.

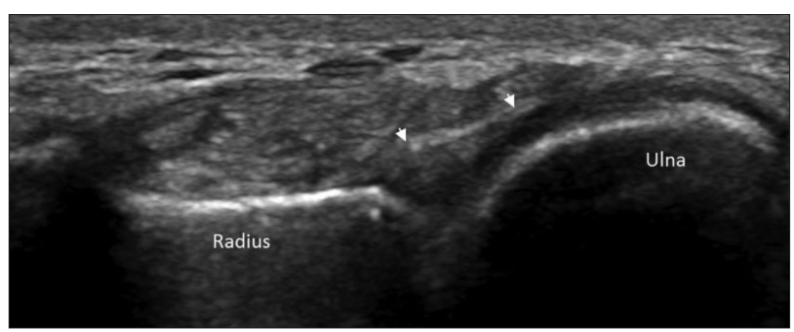

Figure 28. Scapholunate ligament (volar band). The transducer is placed in the transverse plane at the palmar aspect of the distal radius and then moved distally. The volar band of the scapholunate ligament (arrows) is visualized along its long axis as a fibrillar structure connecting the scaphoid and the lunate. It is deeper than the flexor pollicis longus (FPL) tendon.

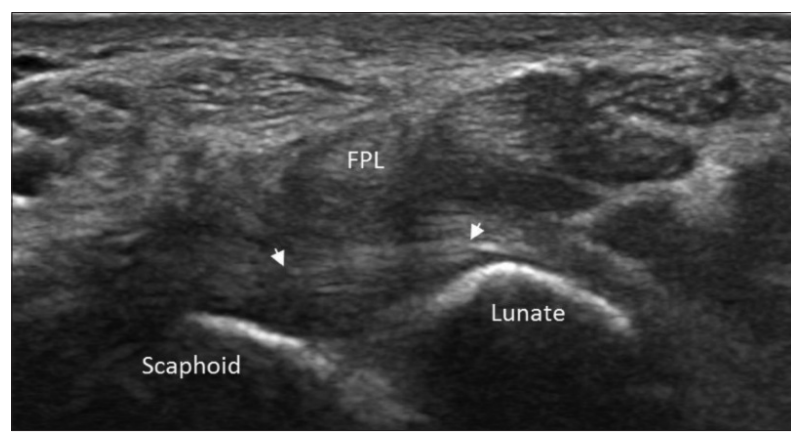




\section{Intracapsular Intrinsic Ligaments}

The intracapsular intrinsic ligaments originate and insert within the carpus and are stronger than the extrinsic ones. They are subdivided into interosseus ligaments, which connect adjacent bones within the proximal or distal carpal row, and midcarpal or intercarpal ligaments, which connect the carpal bones by crossing over the midcarpal joint. ${ }^{12,30}$

The proximal interosseus ligaments are the scapholunate and lunotriquetral ligaments (Figures 28-30) and separate the radiocarpal and midcarpal joint spaces. The distal interosseus ligaments include the trapeziotrapezoid, trapezocapitate, and capitohamate ligaments (Figures 31 and 32) and divide the midcarpal and carpometacarpal joint spaces. ${ }^{6,30}$

Figure 29. Lunotriquetral ligament (volar band). The transducer is placed in the transverse plane at the palmar aspect of the distal radius and then moved distally and translated toward the triquetrum. The volar band of the scapholunate ligament (arrows) is visualized along its long axis as a fibrillar structure connecting the lunate and the triquetrum. The median nerve (MN) within the carpal tunnel and the ulnar nerve (UN) and ulnar artery (UA) within the Guyon canal are also depicted.

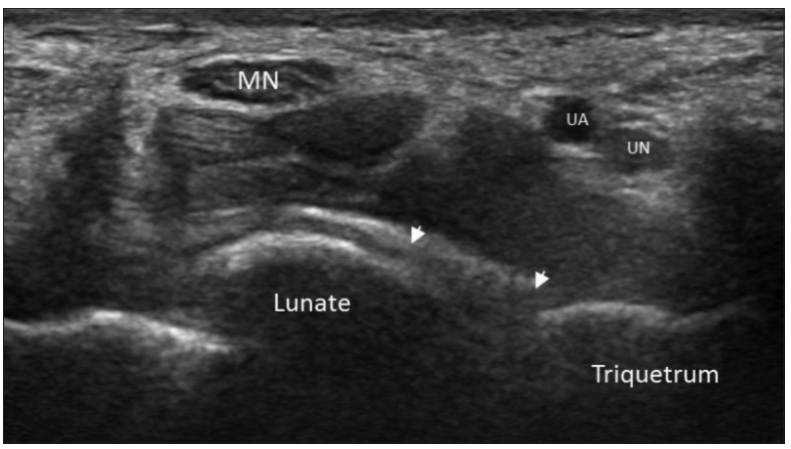

Figure 30. Proximal interosseus ligaments (dorsal bands). The transducer is placed in the transverse plane at the dorsal radius, over the Lister tubercle, and then moved distally toward the dorsal aspect of the first carpal row. The ligaments (arrows) are visualized along their long axis as fibrillar structures extending from the scaphoid to the lunate (scapholunate ligament) and from the lunate and the triquetrum (lunotriquetral ligament).

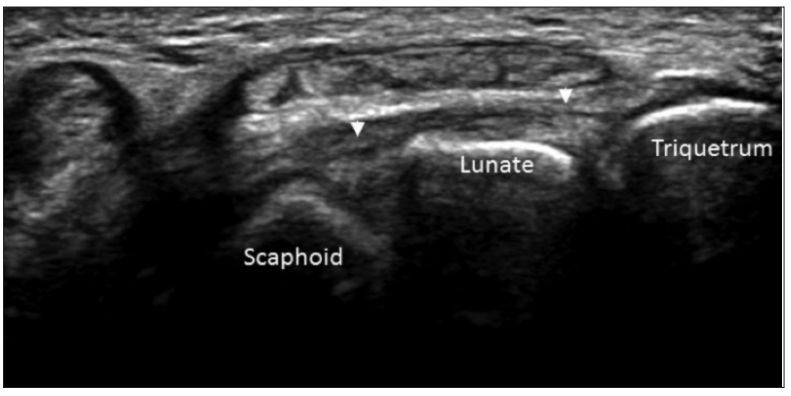

Among the midcarpal ligaments, the dorsal intercarpal and palmar scaphotriquetral ligaments are commonly evaluated by sonography. ${ }^{6}$ The palmar scaphotriquetral ligament (Figure 33) arises from the scaphoid, runs over the capitate without any attachment to it, and inserts into the triquetrum. The dorsal intercarpal ligament (Figure 34), also known as the dorsal scaphotriquetral ligament, originates from the triquetrum and attaches with a proximal band to the scaphoid and with a distal band to the trapezoid. It may have additional insertions into the lunate or trapezium. ${ }^{6,30}$

\section{Conclusions}

The soft tissue structures of the wrist are ideally suited for sonographic assessment. Thanks to the high resolution of latest-generation equipment, tendons, nerves, and ligaments can be fully examined in exquisite detail. Despite the

Figure 31. Distal interosseus ligaments (volar bands). The transducer is placed in the transverse plane at the volar radius and moved distally toward the volar aspect of the first and then the second carpal rows. The ligaments (arrows) are visualized along their long axis as fibrillar structures extending from the trapezium to the trapezoid (trapeziotrapezoid ligament), from the trapezoid to the capitate (trapezocapitate ligament), and from the capitate to the hamate (capitohamate ligament).

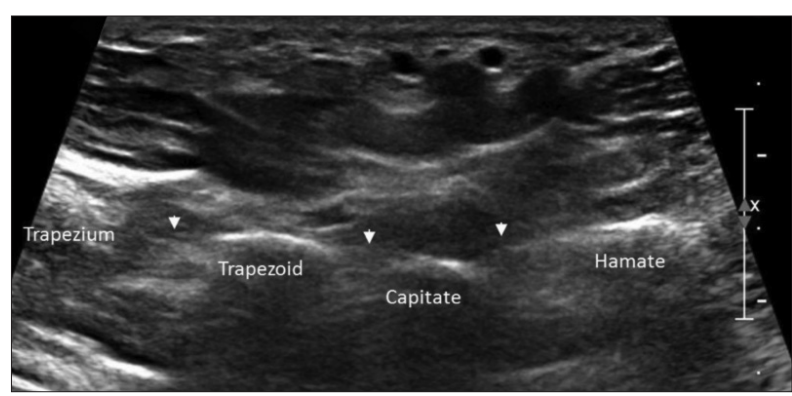

Figure 32. Distal interosseus ligaments (dorsal bands). The transducer is placed in the transverse plane at the dorsal radius, over the Lister tubercle; thereafter, it is moved distally toward the dorsal aspect of the first and then the second carpal rows. The ligaments (arrows) are visualized along their long axis as fibrillar structures extending from the trapezium to the trapezoid (trapeziotrapezoid ligament), from the trapezoid to the capitate (trapezocapitate ligament), and from the capitate to the hamate (capitohamate ligament).

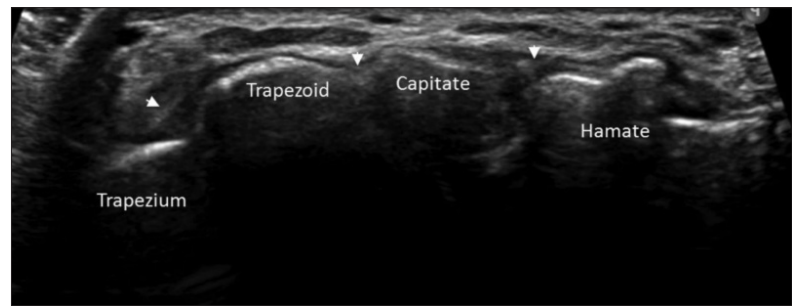


complexity of the wrist joint, a clear understanding of the normal sonographic appearance of the wrist, as well as common variations in its normal anatomy, can then allow a comprehensive evaluation of the soft tissue structures of the wrist.

Figure 33. Palmar scaphotriquetral ligament. The transducer is placed in the transverse plane at the palmar aspect of the distal radius and then moved distally along the first carpal row. The ligament (arrows) is seen along its long axis as a fibrillar structure arising from the scaphoid to the triquetrum. The median nerve (MN) within the carpal tunnel and the ulnar nerve (UN) and ulnar artery (UA) within the Guyon canal are also depicted.

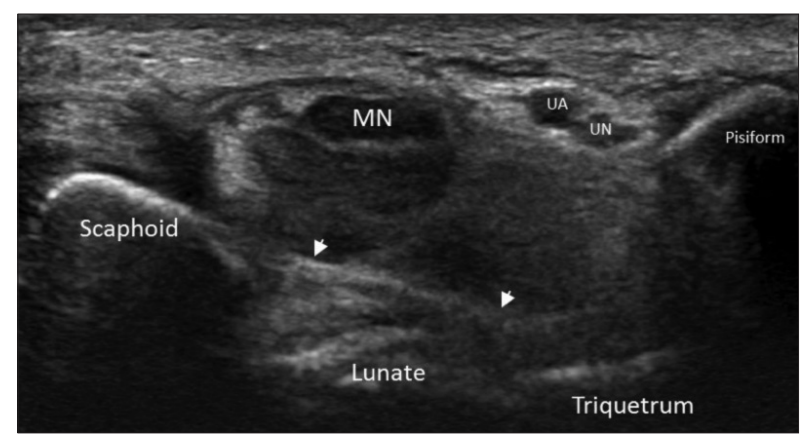

Figure 34. Dorsal intercarpal ligament. The transducer is placed in the transverse plane at the dorsal radius, over the Lister tubercle, and then moved distally along the dorsal aspect of the first carpal row. The proximal band of the ligament (arrows) is visualized along its long axis as a fibrillar structure arising from the scaphoid to the triquetrum, deeper than the fourth extensor compartment $(\mathbf{A})$. Thereafter, the transducer is pointed at the triquetrum and rotated toward the trapezoid. The distal band of the ligament (arrows) is seen along its long axis as a fibrillar structure extending between these 2 bones (B).
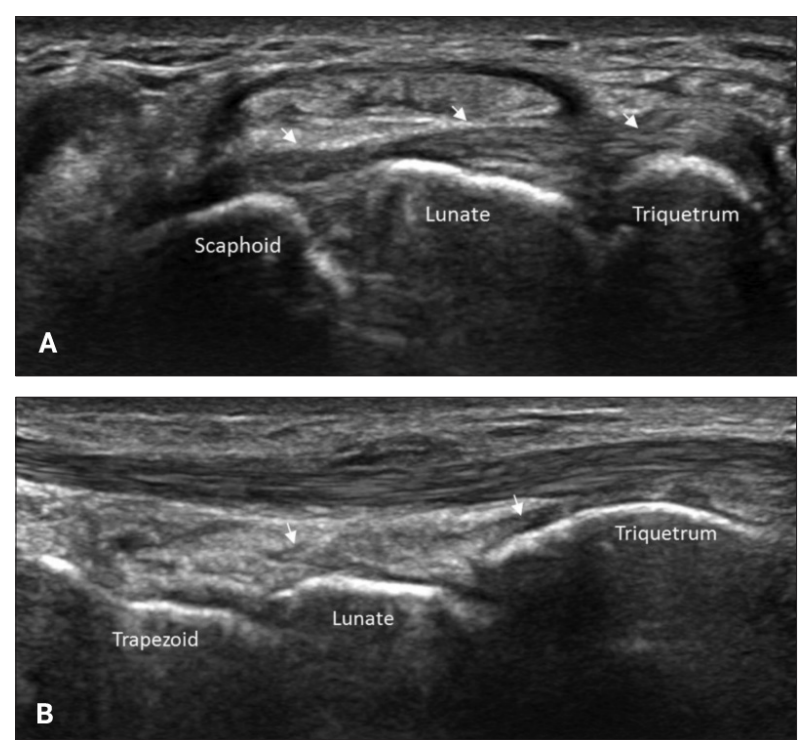

\section{References}

1. Lok RL, Griffith JF, Ng AW, Wong CW. Imaging of radial wrist pain, part II: pathology. Skeletal Radiol 2014; 43:725-743.

2. Sachar K. Ulnar-sided wrist pain: evaluation and treatment of triangular fibrocartilage complex tears, ulnocarpal impaction syndrome, and lunotriquetral ligament tears. J Hand Surg Am 2012; 37:1489-1500.

3. Olubaniyi BO, Bhatnagar G, Vardhanabhuti V, Brown SE, Gafoor A, Suresh PS. Comprehensive musculoskeletal sonographic evaluation of the hand and wrist. J Ultrasound Med 2013; 32:901-914.

4. Sofka CM.Ultrasound of the hand and wrist. Ultrasound Q2014; 30:184-192.

5. American Institute of Ultrasound in Medicine, American College of Radiology, Society for Pediatric Radiology, Society of Radiologists in Ultrasound. AIUM practice guideline for the performance of a musculoskeletal ultrasound examination. J Ultrasound Med 2012;31:1473-1488.

6. Taljanovic MS, Goldberg MR, SheppardJE, Rogers LF. US of the intrinsic and extrinsic wrist ligaments and triangular fibrocartilage complex: normal anatomy and imaging technique. Radiographics 2011;31:e44.

7. Martinoli C, Bianchi S, Dahmane M, Pugliese F, Bianchi-Zamorani MP, Valle M. Ultrasound of tendons and nerves. Eur Radiol 2002; 12:44-55.

8. Draghi F. Ultrasonography of the Upper Extremity: Hand and Wrist. Cham, Switzerland: Springer; 2014.

9. Timins ME, O'Connell SE, Erickson SJ, Oneson SR. MR imaging of the wrist: normal findings that may simulate disease. Radiographics 1996; 16:987-995

10. Boutry N, Lapegue F, Masi L, Claret A, Demondion X, Cotten A. Ultrasonographic evaluation of normal extrinsic and intrinsic carpal ligaments: preliminary experience. Skeletal Radiol 2005; 34:513-521.

11. Lee JC, Healy JC. Normal sonographic anatomy of the wrist and hand. Radiographics 2005; 25:1577-1590.

12. Standring S. Wrist and hand. In: Standring S (ed). Gray's Anatomy: The Anatomical Basis of Clinical Practice. 40th ed. Edinburgh, Scotland: Churchill Livingstone; 2008:857-898.

13. Clavero JA, Golano P, Farinas O, Alomar X, Monill JM, Esplugas M. Extensor mechanism of the fingers: MR imaging-anatomic correlation. Radiographics 2003; 23:593-611.

14. Bianchi S, Martinoli C. Wrist. In: Ultrasound of the Musculoskeletal System. Berlin, Germany: Springer; 2007:425-494.

15. de Lima JE, Kim HJ, Albertotti F, Resnick D. Intersection syndrome: MR imaging with anatomic comparison of the distal forearm. Skeletal Radiol 2004; 33:627-631.

16. Draghi F, Bortolotto C. Intersection syndrome: ultrasound imaging. Skeletal Radiol 2014; 43:283-287.

17. Draghi F, Gregoli B, Bortolotto C. Pisiform bursitis: a forgotten pathology. JClin Ultrasound 2014; 42:560-561.

18. Presazzi A, Bortolotto C, Zacchino M, Madonia L, Draghi F. Carpal tunnel: normal anatomy, anatomical variants and ultrasound technique. J Ultrasound 2011; 14:40-46.

19. Mallinson P, Robinson P. Hand and wrist. In: Beggs I (ed). Musculoskeletal Ultrasound. Philadelphia, PA: Lippincott Williams \& Wilkins; 2013 73-102. 
20. Schmidt HM. The "loge de Guyon": a contribution to the clinical anatomy of the human hand. Acta Anat 1988; 131:113-121.

21. Sookur PA, Naraghi AM, Bleakney RR, Jalan R, Chan O, White LM. Accessory muscles: anatomy, symptoms, and radiologic evaluation. Radiographics 2008; 28:481-499.

22. AlbrightJA, Linburg RM. Common variations of the radial wrist extensors. J Hand Surg Am 1978; 3:134-138.

23. Nayak SR, Krishnamurthy A, Prabhu LV, Rai R, Ranade AV, Madhyastha S. Anatomical variation of radial wrist extensor muscles: a study in cadavers. Clinics (Sao Paulo) 2008; 63:85-90.

24. Pai MM, Prabhu LV, Nayak SR, et al. The palmaris longus muscle: its anatomic variations and functional morphology. Rom J Morphol Embryol 2008; 49:215-217.

25. Reimann AF, Daseler EH, Anson BJ, Beaton LE. The palmaris longus muscle and tendon: a study of 1600 extremities. Anat Rec 1944; 89:495505.

26. Zeiss J, Guilliam-Haidet L. MR demonstration of anomalous muscles about the volar aspect of the wrist and forearm. Clin Imaging 1996; 20:219-221.

27. Rodriguez-Niedenfuhr M, Vazquez T, Golano P, Parkin I, Sanudo JR. Extensor digitorum brevis manus: anatomical, radiological and clinical relevance - a review. Clin Anat 2002; 15:286-292.

28. Pierre-Jerome C, Smitson RD Jr, Shah RK, Moncayo V, Abdelnoor M, Terk MR. MRI of the median nerve and median artery in the carpal tunnel: prevalence of their anatomical variations and clinical significance. Surg Radiol Anat 2010; 32:315-322.

29. TaleisnikJ. The ligaments of the wrist. J Hand Surg Am 1976; 1:110-118.

30. Berger RA. The anatomy of the ligaments of the wrist and distal radioulnar joints. Clin Orthop Relat Res 2001; 383:32-40.

31. Lacelli F, Muda A, Sconfienza LM, Schettini D, Garlaschi G, Silvestri E. High-resolution ultrasound anatomy of extrinsic carpal ligaments. Radiol Med 2008; 113:504-516.

32. Theumann NH, Pfirrmann CW, Antonio GE, et al. Extrinsic carpal ligaments: normal MR arthrographic appearance in cadavers. Radiology 2003; 226:171-179.

33. Taleisnik J. Current concepts review: carpal instability. J Bone Joint Surg Am 1988; 70:1262-1268.

34. Taljanovic MS, Malan JJ, Sheppard JE. Normal anatomy of the extrinsic capsular wrist ligaments by 3-T MRI and high-resolution ultrasonography. Semin Musculoskelet Radiol 2012; 16:104-114.

35. Berger RA, Kauer JM, Landsmeer JM. Radioscapholunate ligament: a gross anatomic and histologic study of fetal and adult wrists. J Hand Surg Am 1991; 16:350-355.

36. Shahabpour M, De Maeseneer M, Pouders C, et al. MR imaging of normal extrinsic wrist ligaments using thin slices with clinical and surgical correlation. Eur J Radiol 2011; 77:196-201.

37. Stein JM, Cook TS, Simonson S, Kim W. Normal and variant anatomy of the wrist and hand on MR imaging. Magn Reson Imaging Clin North Am 2011; 19:595-608. 